\title{
ISOLAMENTO E IDENTIFICAÇÃO DE FUNGOS PRESENTES EM PELOS DE CÃES HÍGIDOS E COM SINTOMAS DE DERMATOFITOSE, DO MUNICÍPIO DE ARAÇATUBA, SÃO PAULO
}

\author{
Natália Tonheiro Cardoso¹, Danila Fernanda Rodrigues Frias², Dora Inés Kozusny- \\ Andreani ${ }^{1}$ \\ 1 UNICASTELO \\ 2 EMBRAPA - GENEPLUS \\ Correspondência: Danila Fernanda Rodrigues Frias: danilafrias@yahoo.com.br
}

RESUMO: A dermatofitose é uma doença de caráter zoonótico causada por fungos patogênicos chamados dermatófitos. O objetivo deste trabalho foi identificar os fungos presentes em pelos de cães hígidos e com sintomas de dermatofitose do Município de Araçatuba, São Paulo. Foram coletadas 100 amostras de pelos, destas 50 foram provenientes de cães aparentemente hígidos coletadas em clínicas veterinárias e pet shop, e 50 provenientes de cães com sintomas de dermatofitose coletadas no centro de controle de zoonoses. Em cada grupo, 25 amostras eram de machos e 25 de fêmeas. Para detecção do fungo, foi realizado exame direto nos pelos através da clarificação com hidróxido de potássio a $20 \%$ e observação ao microscópio. Também foi realizado cultivo em Placas de Petri, com posterior identificação macroscópica e microscópica dos agentes. Nos dois grupos, todas as amostras foram positivas. Nos animais hígidos, o fungo mais encontrado foi o Penicillium spp, seguido pelo dermatófito Microsporum canis. Nos animais com sintomas de dermatofitose, o fungo mais isolado também foi o Penicillium spp, mas tiveram grande destaque os dermatófitos Microsporum canis e Trichophyton mentagrophytes. De acordo com os resultados obtidos conclui-se que os cães são reservatórios naturais de fungos sapróbios e dermatófitos mesmo sem apresentar lesão aparente na pele. Em nenhum grupo ouve diferença entre os sexos e o dermatófito de maior ocorrência foi o Microsporum canis.

Palavras-chave: dermatófitos; sapróbios; Microsporum canis; Penicillium spp; Trichophyton mentagrophytes

\section{ISOLATION AND IDENTIFICATION OF FUNGI PRESENT IN HEALTHY DOGS' HAIR AND IN DOGS WITH SYMPTOMS OF DERMATOPHYTOSIS IN THE CITY OF ARAÇATUBA, SÃO PAULO}

\begin{abstract}
The Dermatophytosis (Ringworm) is a zoonotic disease caused by pathogenic fungi called dermatophytes. The aim of this study was to identify fungi present in healthy dogs hair and with symptoms of dermaphytosis in Araçatuba, São Paulo. One hundred of hair samples were collected, 50 of these from apparently healthy dogs collected in veterinary clinics and pet shops, and 50 from dogs with symptoms of dermatophytosis collected on zoonosis control centers. In each group, 25 samples were from males and 25 from females. For the detection of fungi, an examination direct in hair was conducted by clarifying with potassium hydroxide $20 \%$ and observation under a microscope. It was also performed culture, with Petri dishes with further macroscopic and microscopic identification of agents. In both groups, all samples were positive. In healthy animals, the most found fungus was Penicillium spp., followed by the dermatophyte Microsporumcanis. In animals with symptoms of dermatophytosis, the most isolated fungus was also the Penicillium spp., but dermatophytes Microsporumcanis and Trichophyton mentagrophytes had great prominence. According to the results, it was concluded that the dogs are natural reservoirs of saprobic fungus and dermatophyte even without apparent injury in the skin. There was no difference between sexes and the highest occurrence was the dermatophyte Microsporumcanis.
\end{abstract}

Key Words: dermatophytes; saprobe; Microsporum canis; Penicillium spp; Trichophyton mentagrophytes 


\section{INTRODUÇÃO}

A pele é considerada o maior órgão do corpo, sendo responsável pela proteção de todo organismo. As alterações neste órgão são avistadas quando já estão evidentes, como por exemplo, a perda de pelos, o início do prurido, alterações no odor da pele, alteração na pigmentação, presença de descamações ou crostas, sendo estes os principais sinais de apresentação de doença dermatológica, associados à sensibilidade ao toque (Wilkinson \& Harvey, 1995).

Dermatofitoses são micoses superficiais causadas por fungos denominados dermatófitos, pertencentes ao grupo dos fungos filamentosos, hialinos, septados, queratinofílicos, sensíveis à griseofulvina, capazes de colonizar e causar lesões no extrato córneo do homem e animais (Mendleau \& Hnilica, 2003; Brilhante et al., 2004). É uma doença de caráter contagioso também conhecida como "tinha" ou ringworm, que apresenta marcada afinidade pela queratina afetando, portanto, pelos, unhas, cascos, penas e células queratinizadas da pele (Thadei, 2012).

Os dermatófitos são classificados em três gêneros: Epidermophyton, Microsporum e Trichophyton, que incluem cerca de 40 espécies, das quais somente algumas, pertencentes aos gêneros Microsporum e Trichophyton são usualmente as causas de dermatofitose em animais domésticos. Podem ser divididos em três grupos ecológicos, conforme seu habitat e/ou hospedeiros naturais, em antropofílicos (humanos), zoofílicos (animais) e geofílicos (solo) (Cabañes, 2000).

De acordo com Thadei (2012), os dermatófitos infectam várias espécies animais, determinando de modo geral, uma área circular de perda de pelo com a borda externa alta e avermelhada, pele escurecida e a face, as orelhas, as patas e a cauda são as áreas geralmente mais afetadas. Estas lesões são resultado de uma reação inflamatória ao fungo, provocando lesões secas e, comumente, não pruriginosas que se distribuem focalmente na superfície cutânea, sem causar transtornos gerais aos animais afetados (Jungerman \& Schwartzman, 1972; Pereira \& Meireles, 2000; Baranová et al., 2003).

Dentre os dermatófitos, o Microsporum canis é o fungo que acomete cães e gatos com mais frequência. A sua transmissão ocorre pelo contato de pelos e escamas infectadas ou do próprio fungo presente nos animais, ambiente, escovas, pentes, etc. Animais jovens, idosos e com deficiência imunológica são acometidos com mais frequência podendo ser fonte de infecção para outros animais ou até para o homem (Bichard \& Sherding, 2008).

O diagnóstico da dermatofitose baseia-se na evidência da infectividade, na aparência das lesões características e na presença de micélios ou esporos do fungo (Harvey \& Mckeever, 2004). O exame microscópico direto de pelos infectados tem valor de diagnóstico, porém o método mais comumente empregado e mais confiável para 0 diagnóstico da dermatofitose é o uso de culturas fúngicas (Feitosa, 2008).

Outra técnica empregada no diagnóstico é atraves da utilização de uma lâmpada especial, conhecida como lâmpada de Wood, que detecta a presença de dermatófito. Infelizmente, apenas $50 \%$ de um tipo específico de fungo, chamado Microsporum canis fica fluorescente no pelo do animal, por isso, um resultado negativo com a utilização da lâmpada de Wood não descarta a possibilidade da presença de dermatófitos (Harvey \& Mckeever, 2004).

Para que o tratamento dessas afecções seja efetivo, o diagnostico do 

de dermatofitose, do município de Araçatuba, São Paulo

gênero e espécie causal da doença é essencial, pois para cada tipo e agente, existem fármacos mais eficientes (Guaguère \& Bensignor, 2005).

Devido a todos os fatores já discutidos, o presente trabalho teve por objetivo diagnosticar os agentes etiológicos das dermatofitoses em cães com sintomas sugestivos de dermatofitose e cães hígidos do Município de Araçatuba, SP.

\section{MATERIAL E MÉTODOS}

O experimento foi conduzido no Laboratório de Microbiologia, da Universidade Camilo Castelo Branco, Campus Fernandópolis, SP. Durante o período entre Fevereiro a Maio de 2012, foram coletadas amostras de pelos de 100 cães, da cidade de Araçatuba-SP, distribuídos em dois grupos: a) 50 animais hígidos, provenientes de clínicas e pet shop; b) 50 animais com sintomas de dermatofitose do Centro de Controle de Zoonoses do Município. Em ambos os grupos $50 \%$ dos animais eram machos e $50 \%$ fêmeas.

As amostras de pelos foram retiradas com pinça estéril, depositadas em coletores universais estéreis com tampas e transportados para 0 laboratório. Parte do material foi utilizado para exame microscópico direto. Para tal finalidade os pelos foram depositados em lâminas e clareados pela adição de solução de hidróxido de potássio a $20 \%$ com leve aquecimento. Em seguida, o material foi observado ao microscópio óptico (40x) para verificação de estruturas fúngicas (artrósporos).

O restante do material foi cultivado em Ágar Sabourau e Ágar Sabouraud enriquecido com extrato de levedura, tiamina, antibióticos (estreptomicina e cloranfenicol) e cicloheximida. Este último composto serve para supressão de crescimento de fungos saprofíticos que possam inibir o desenvolvimento dos agentes etiológicos da dermatofitose. A microbiota saprofítica se desenvolve mais rapidamente impossibilitando 0 isolamento dos dermatofitos.

As Placas de Petri contendo os diferentes meios de cultura foram inoculadas e incubadas a $34^{\circ} \mathrm{C}$ por 10 dias, sendo avaliadas a partir do terceiro dia de incubação para verificação do estabelecimento da cultura, no entanto o diagnóstico foi realizado no décimo dia.

A avaliação foi conduzida de acordo com as características macroscópicas e microscópicas. O exame microscópico foi realizado utilizando a técnica descrita por Minami (2003). Adicionou-se em uma lâmina uma gota de lactofenol azul-algodão. Em seguida, foi retirado um fragmento das bordas da cultura sendo depositado sobre a gota de corante, e posteriormente foi depositada uma lamínula. Após este procedimento as estruturas foram observadas ao microscópio óptico (40x). Para identificação macroscópica foram avaliadas as características das colônias levando em consideração o anverso e reverso das mesmas, como descrito por De la Maza et al. (1999) e Minami (2003).

Os dados obtidos foram submetidos à análise de variância e as médias foram comparadas pelo teste de Tukey com $5 \%$ de probabilidade, utilizando software Assitat version 7.6 beta (Silva \& Azevedo, 2009).

\section{RESULTADOS E DISCUSSÃO}

O exame direto com a utilização da técnica de clarificação com hidróxido de potássio favoreceu a visualização de estruturas de parasitismo, localizados na parte externa e na parte interna dos pelos. Quando os dermatófitos são encontrados fora do pelo, o parasitismo 
se denomina ectotrix e, dentro do pelo o parasitismo é chamado endotrix (Minami, 2003; Quinn et al., 2005).

A positividade das culturas foi verificada em $100 \%$ dos animais com sintomatologia de dermatofitose, sem apresentar diferenças quanto ao sexo. Brilhante et al. (2003) e Pinter \& Stritof (2004), não encontraram diferenças quanto achados fungicos em amostras avaliadas de animais de sexos e raças diferentes. A Tabela 1 mostra as proporções de fungos patogênicos isolados de pelos de animais doentes e hígidos.

Tabela 1: Proporção individual de dermatofitos e fungos saprofíticos isolados de
pêlos de cães com sintomas de dermatofitose e hígidos. Araçatuba, São
Paulo, Brasil.

O dermatófito Microsporum canis foi isolado na maioria das amostras dos animais doentes sem diferença entre os sexos. Os resultados obtidos neste trabalho confirmam os encontrados por outros autores, que identificaram $M$. canis como 0 dermatófito mais frequentemente isolado em animais domésticos (Brilhante et al., 2003; Bernardo et al., 2005; Frias \& KozusnyAndreani, 2008; Ramadinha et al., 2010).

Verificou-se que os pelos dos cães doentes e acometidos por $M$. canis, machos e fêmeas apresentaram parasitismo ectotrix, com presença de esporos arredondados, pequenos, formando uma bainha ao redor do pelo. Esta forma de parasitar o pelo é característica do gênero Microsporum. Os pelos infectados por Microsporum apresentam acúmulo de esporos na superfície externa e produzem fluorescência esverdeada oriunda de um metabólito do triptofano que é visível quando exposto a luz ultravioleta (Quinn et al., 2005).

Este dermatofito também foi isolado das amostras provenientes de animais aparentemente hígidos, mostrando que os cães são portadores sãos deste fungo. Este fato é importante quando relacionamos os casos de dermatofitoses humanas, onde a maioria também é causada por este tipo de fungo (Copetti et al., 2006). Isso pode ser explicado devido ao estreito relacionamento entre estas espécies.

Por outro lado, o M. gypseum, classificado como fungo geofílico, foi isolado em numero elevado nos animais com sintomas de dermatofitose, em $32 \%$ das fêmeas e $34 \%$ dos machos, evidenciando diferenças significativas com os animais hígidos (Tabela1). Este fungo é considerado como agente pouco frequente nas dermatofitoses de animais domésticos, inclusive não se conhecem surtos interespecíficos determinados por este agente (Costa et al., 1994). Kozak et al. (2003) em estudos sobre dermatofitose em cães não isolaram $M$. gypseum. No entanto Coelho et al. (2008) encontraram este dermatofito em 15,8\% das amostras clínicas analisadas, e Frias \& Kozusny-Andreani (2008) isolaram em $26 \%$ das amostras.

$$
\text { O fungo Trichophyton }
$$
mentagrophytes foi isolado em machos $(52 \%)$ e fêmeas $(48 \%)$ que apresentaram sinais clínicos de dermatofitose, sendo também encontrado em animais hígidos, porém em menor proporção. Outros autores também citaram a ocorrência deste fungo em pelos de animais hígidos, em menores proporções (Frias \& KozusnyAndreani, 2008).

Os fungos saprofíticos encontrados foram dos gêneros 

de dermatofitose, do município de Araçatuba, São Paulo

Penicillium, Rhizopus, Aspergillus e Fusarium tanto em animais com sinais clínicos de dermatofitose como nos hígidos. Este resultado corrobora com os encontrados por Paixão et al. (2001) e Frias \& Kozusny-Andreani (2008).

Foram encontradas associações entre fungos saprofíticos e dermatófitos. Estas associações são bastante frequentes, e os resultados obtidos neste trabalho são semelhantes aos descritos por Paixão et al. (2001), Frias \& Kozusny-Andreani (2008) e Coelho et al. (2008).

Tanto os animais machos como as fêmeas com sinais clínicos de dermatofitose apresentaram culturas positivas em meio Agar Sabouraud para Candidas albicans e Malassezia spp. Kozak et al. (2003) isolou C. albicans e Malassezia em 5\% e 54\%, dos animais avaliados, respectivamente.

isolamento de Malassezia em material proveniente de tegumento e pelos de cães é frequente devido a serem comensais de pele de animais, e a colonização e o crescimento deste micro-organismo podem estar associados à imunossupressão e a outros fatores predisponentes (Quinn et al., 2005).

\section{CONCLUSÃO}

De acordo com os resultados obtidos nas condições em que foi realizado 0 experimento pode-se concluir que os cães são reservatórios de fungos dermatófitos e sapróbios, já que as amostras coletadas de cães sem nenhuma lesão de pele visível, isolaram-se estes fungos. Por este motivo, e pela dermatofitose ser uma zoonose, o contato entre o ser humano e seus animais de estimação deve ser criterioso, principalmente crianças e pessoas imunossuprimidas, devido a real hipótese de adquirir o fungo e desenvolver a doença.
Não houve diferença no isolamento de fungos sapróbios entre animais de diferentes sexos tanto no grupo de animais hígidos como no de com sintomas de dermatofitose. A maior frequência de isolamento nos animais com sintomas de dermatofitose, no município de Araçatuba, foi do dermatófito Microsporum canis.

\section{REFERÊNCIAS}

BILEK, J.; BARANOVA, Z., KOZAK, M. et al. Trichophyton mentagrophytes var. quinckeanum as a cause of zoophilic dermatomycosis in a human family. Bratisl Lek Listy, v.106, n.12, p.383-385, 2005.

BERNARDO, F.; LANÇA, A.; GUERRA, M. M. Dermatofitos isolados de animais de companhia (cão e gato), em Lisboa, Portugal (2002-2004). Revista Portuguesa de Ciência Veterinária, v.100, p.85-88, 2005.

BICHARD, S. J.; SHERDING, R. G. Clínica de pequenos animais: Manual Saunders. 3. ed. São Paulo: Roca, 2008. p. 458- 459.

BRILHANTE, R. S. N.; CAVALCANTE, C. S. P.; SOARES-JUNIOR, F. A. et al. High rate Microsporum canis feline and canine dermatophytoses in Northeast Brazil: epidemiological and diagnosis features. Mycopathologia, v.156, n.4, p.303-308, 2003.

BRILHANTE, R. S. N.; CAVALCANTE, C. S. P.; SOARES-JUNIOR, F. A. et al. Evaluation of Microsporum canis in diferente methods of storage. Medical Mycology, v.42, n.6, p.499504, 2004.

CABAÑES, F. J. Dermatofitosis animales. Recientes avances. Revista Iberoamericana de Micologia, v.17, n.1, p.S8-S12, 2000.

COELHO, A. C.; LEGRIA, N.; RODRIGUES, J. Isolamento de dermatofitos em animais domésticos em Vila Real, Portugal. Arquivo Brasileiro de Medicina Veterinária e Zootecnia, v.60, n.4, p.1017-1020, 2008.

COPETTI, M. V.; SANTURIO, J. M.; CAVALHEIRO, A. S. et al. Dermatophytes isolated from dogs and cats suspected of dermatophytosis in Southern Brazil. Acta Scientiae Veterinariae, v.34, n.2, p.119-124, 2006. 
COSTA, E. O; DINIZ, L. S. M.; BENITES, N. R. et al. Surtos interespecíficos por Microsporum canis e Microsporum gypseum. Revista de Saúde Publica, v.8, n.5, p.337-340, 1994.

DE LA MAZA, L. M.; PEZZLO, M. T.; BARON, E. J. Atlas de diagnóstico em microbiologia. Ed Artmed. 1999, 216p.

FEITOSA, F. L. F. Semiologia Veterinária: A arte do diagnóstico. 2. ed. São Paulo: Roca, 2008. p. 609.

FRIAS, D. F. R.; KOZUSNY-ANDREANI, D. I. Isolamento de dermatofitos e fungos sapróbios de pelos de cães de Fernandópolis, Brasil. CES, Veterinária e Zootecnia, v.2, p.52-60, 2008.

GUAGUÈRE, É.; BENSIGNOR, E. Terapêutica dermatológica do cão. São Paulo: Roca, 2005. p. 196-199.

HARVEY, R. G.; MCKEEVER, P. J. Manual colorido de dermatologia do cão e do gato: Diagnóstico e Tratamento. Rio de Janeiro: Revinter, 2004. p. 210-212.

JUNGERMAN, P. F.; SCHWARTZMAN, R. M. Veterinary Medical Mycology. Philadelphia: Lea \& Febiger, 1972. p.3-28.

KOZAK, M.; BILEK, J.; BELADICOVA, K. et al. Study of the dermatophytes in dogs and the risk of human infection. Bratisl Lek Listy, v.104, n.7-8, p.211-217, 2003.

MENDLEAU, L.; HNILICA, K. A. Dermatologia de pequenos animais: Atlas colorido e guia terapêutico.1. ed. Roca, 2003.

MINAMI, P. S. Micologia. Métodos laboratoriais de diagnósticos das micoses. Ed. Manole, 2003, 199p.

PAIXÃO, G. G.; SIDRIM, M. J. J. C.; CAMPOS, G. M. M. et al. Dermatófitos e fungos sapróbios isolados de cães e gatos. Fortaleza. 2001. p.53.

PEREIRA, D. B.; MEIRELES, M. C. As doenças causadas por fungos e oomycetos.

Dermatofitoses. In: RIET CORREA, F.; SCHILD, A. L.; MÉNDEZ, M. C. et al. Doenças de ruminantes e eqüinos. 2. ed. São Paulo: Varela, 2000. p.367-373.

PINTER, I.; STRITOF, Z. A retrospective study Trichophyton metantagrophytes infection in dogs
(1970-2002). Veteririnars kiarhiv, v.74, n.4, p.251-260, 2004.

QUINN, P. J.; MARKEY, B. K.; CARTER, M. E. et al. Microbiologia Veterinária e Doenças Infecciosas. Porto Alegre: Artmed, 2005.

RAMANDINHA, R. R.; REIS, R. K.; CAMPOS, S. G. et al. Lufenuron no tratamento da dermatofitose em gatos. Pesquisa Veterinária Brasileira, v.30, n.2, p.132-138, 2010.

SILVA, F. de A. S.; AZEVEDO, C. A. V. de. Principal componentes analysis in the software Assitat-Statistical attendance. In: World Congresso Computers in Agriculture, 7, Reno-NV-USA: American Society of Agricultural and Biological Engineers, 2009.

THADEI, C. L. Dermatofitoses (Fungos dermatófitos). Disponível em <http://www.saudeanimal.com.br/artig137.htm> Acesso em: 17 jul. 2012.

WILKINSON, G. T.; HARVEY, R. G. Atlas colorido de dermatologia dos pequenos animais. 2. ed. São Paulo: Manole, 1995. p.215, 207. 